\title{
Superoxide Dismutase
}

National Cancer Institute

\section{Source}

National Cancer Institute. Superoxide Dismutase. NCI Thesaurus. Code C17172.

Proteins that protect cells against elevated levels of superoxide a reactive oxygen species. These antioxidant enzymes catalyze the formation of molecular oxygen and hydrogen peroxide from the superoxide anion. They are also potential therapeutic agents in diseases related to oxidative stress and in the regulation of the aging process. 